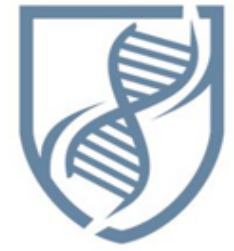

Journal of Bioscience and Applied Research
JBAAR

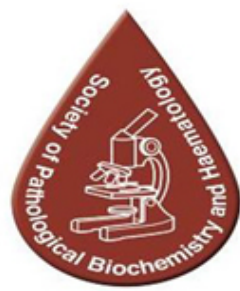

WWW.JBAAR.ORG

\title{
Efficacy of a novel water soluble curcumin derivative versus Tadalafile in mediating erectile function
}

\author{
Zaahkouk,SA.M. ${ }^{1}$, Atta H.M ${ }^{2}$., Fouad Hanan. ${ }^{2}$, Hesham G.A ${ }^{1}$. Yehia M. Hussein. ${ }^{1}$ \\ ${ }^{1}$ Zoology Department, Faculty of Science, Al-Azhar University \\ ${ }^{2}$ Medical Biochemistry Department, Faculty of Medicine, Cairo University
}

\begin{abstract}
The present study was conducted to assess the efficacy of a novel curcumin derivative (NCD) versus Tadalafile in erectile signaling. The study was conducted on 15 control male rats and 75 diabetic male rats divided into the following groups:, diabetic, curcumin, NCD, Tadalafile and NCD combined with Tadalafile . Cavernous tissue gene expression levels of heme oxygenase-1 (HO-1), Nrf2, $\mathrm{NF}-\beta$, and $\mathrm{p} 38$, enzyme activities of heme oxygenase (HO) and nitric oxide synthase (NOS), cGMP and intracavernosal pressure (ICP) were assessed. Results showed that 12 weeks after induction of diabetes, erectile dysfunction (ED) was confirmed by the significant decrease in ICP, a significant decrease in cGMP, NOS, HO enzyme activities, a significant decrease in HO-1 gene and a significant elevation of $\mathrm{NF}-\beta, \quad$ p38 genes. Administration of all therapeutic interventions led to a significant elevation in ICP, cGMP levels, a significant increase in HO-1 and NOS enzymes, a significant increase in $\mathrm{HO}-1$, and $\mathrm{Nrf} 2$ gene expression, and a significant decrease in NF $-\beta$, p38 gene expression. NCD or its combination with Tadalafile showed significant superiority and more prolonged duration of action. In conclusion, NCD could enhance erectile function with more efficacy and more prolonged duration of action.
\end{abstract}

Keywords: Erectile dysfunction, cGMP, HO-1, Nrf2, p38, NF $-\beta$, NOS, Rats.

\section{Introduction}

Diabetes mellitus (DM) is a metabolic disorder resulting from a defect in insulin secretion, insulin action, or both. Insulin deficiency in turn leads to chronic hyperglycemia with disturbances of carbohydrate, fat and protein metabolism (Lindberg et al., 2012). Diabetes is the most common endocrine disorder and it was reported that by the year 2010 more than 200 million people worldwide will have DM and 300 million will subsequently have the disease by 2025 (King et al.,1998). Most cases of diabetes mellitus fall into the three broad categories of type 1 , type 2 and gestational diabetes. A few other types are described. In the Indian subcontinent and Southeast Asia, turmeric has traditionally been used as a treatment for inflammation, skin wounds, and tumors. Clinical activity of curcumin has yet to be confirmed; however, in preclinical animal models, curcumin has shown cancer chemopreventive, antineoplastic, and anti-inflammatory properties (Hatcher et al ., 2008). Turmeric extract (curcumin) has been shown to improve blood flow as well as strengthen blood vessels and ability to neutralize free radicals, chemicals that could damage cells. Turmeric extract (curcumin) can ward off cancer and tumorous growths (Aggarawl et al ., 2003).

Stimulation of insulin release and lowering blood glucose level in type II diabetes (Seo et al ., 2008). Erectile response depends on nitric oxide (NO) generated by NO synthase (NOS) enzyme of the nerves and vascular endothelium in the cavernous tissue. A role similar to that of NOS/NO signaling was proved for carbon monoxide (CO) produced by heme oxygenase (HO) enzyme (Abdel Aziz et al.,2009). Interestingly, hydrogen sulfide (H2S) a known vasodilator and smooth muscle relaxant was proved to be an inducer for HO-1 gene expression (Hua et al.,2013, D'Araio . et al., 2013). Moreover, (Abdel aziz et al., 2008). reported that the effect of sildenafil, verdenafil and tadalafil are partially mediated via upregulation of $\mathrm{HO}$ enzyme activity and cGMP and their effect is partially inhibited by HO inhibitor. The present study was conducted to compare the molecular and physiological effects of a novel water-soluble curcumin protein conjugates (NCD) 
versus natural curcumin and Tadalafile in experimental diabetic model of erectile dysfunction (ED).

\section{Materials and Methods}

The novel curcumin conjugate is registered as international patent protected by the rights of "The Patent Cooperation Treaty" under: (PCT/EG2010/000008, Published Patent Pending, WO 2011/100984) and is the personal property of its inventors(Jin-Jia Hu et al., 2008). A total number of 90 adult male white albino rats weight (180-200 g) were used in the current study after the approval of the Institutional Animal Care and Use Committee (IACUC).

Experimental diabetes was induced by a single intra peritoneal injection of $65 \mathrm{mg} / \mathrm{kg}$ body weight of streptozotocin (STZ) (Paget et al., 1964). The treatment phase of the study lasted twelve weeks according to the findings reported by ( $\mathrm{Li}$ et al., 2011) who stated that erectile dysfunction is established 12 weeks after diabetic induction in rats. Curcumin dose was chosen according to our previous studies (Abdel Aziz et al.,2007, Zhang et al., 2013). Erectile dysfunction was proved by assessment of intracavernosal pressure (ICP) / mean arterial pressure (MAP) as a physiological index of erectile function. All drug interventions were initiated after 12 weeks of diabetes induction. Animals involve 15 albino male rats served as control group and 75 STZ-induced diabetic rats that were equally divided into the following groups: untreated Diabetic control group, Curcumin group which received $10 \mathrm{mg} / \mathrm{kg}$ body weight (B.Wt.) oral dose of natural curcumin, NCD group which received $2 \mathrm{mg} / \mathrm{kg}$ B.Wt. single oral dose of NCD, Tadalafile group which received Tadalafile citrate dissolved in distilled water (4 $\mathrm{mg} / \mathrm{kg}$; equivalent to $50-\mathrm{mg}$ dose in $70-\mathrm{kg}$ adult man according to Paget's table of experimental studies), rat group that received NCD combined with Tadalafile (NCD+ Tadalafile group) (Paget et al., 1964). Animals were euthanized after $1 \mathrm{hr}, 24 \mathrm{hr}$ and 5 days following drug intervention regimens. cavernous tissue cut into small pieces and homogenate in lyses buffer, samples were kept on crushed ice all times during the preparation then kept frozen at $-70^{\circ} \mathrm{c}$ till analysis. The following parameters were assessed in the cavernous tissue: HO enzyme activity (Abdel Aziz et al., 2008)' NOS activity (Moshag et al., 1995)., cGMP, intracavernosal pressure (ICP) as physiologic assessment of erectile function, and gene expression of $\mathrm{HO}-1$, Nrf2, NF - B, and p38 gene by quantitative real time PCR (Paget et al.,1964, Abdel Aziz et al., 2012).

\section{Results}

\section{a. Change in HO-1 gene}

Results showed that administration of either natural curcumin, NCD, Tadalafile, or NCD\& Tadalafile combination led to a significant elevation in HO-1 gene expression after $24 \mathrm{hr}$ persisting up to 5 days (with natural curcumin, NCD, NCD combination with Tadalafile) with more significant elevation with NCD administration in comparison to natural curcumin and Tadalafile rat groups.
Combined NCD with Tadalafile showed significant enhancing effect when compared to Tadalafile alone. (Tables 1\&2).

Table (1): HO-1 Gene in rats treated with curcumin, tadalafil after injection with streptozotocinat different periods:

\begin{tabular}{|c|c|c|c|c|c|c|c|c|}
\hline Proups & $\begin{array}{l}\text { Mean } \\
\pm \text { SD }\end{array}$ & Control & DM & $\begin{array}{l}\text { DM+ } \\
\text { p.Curc. }\end{array}$ & $\begin{array}{l}\text { DM+ } \\
\text { Curc.D. }\end{array}$ & $\begin{array}{l}\text { DM+ } \\
\text { Znpp+ } \\
\text { Curc.D }\end{array}$ & $\begin{array}{l}\text { DM+ } \\
\text { Tada. }\end{array}$ & $\begin{array}{l}\text { DM+ } \\
\text { Tada.+ } \\
\text { Curc.D }\end{array}$ \\
\hline $\begin{array}{l}\text { HO-1 } \\
\text { gene } \\
(1.5 \mathrm{H})\end{array}$ & $\begin{array}{l}\text { Mean } \\
\pm \mathrm{SD}\end{array}$ & $\begin{array}{l}0.154 \mathrm{a} \\
\pm 0.05\end{array}$ & $\begin{array}{r}0.14 \mathrm{a} \\
\pm 0.02\end{array}$ & $\begin{array}{l}0.16 \mathrm{~b} \\
\pm 0.05\end{array}$ & $\begin{array}{l}0.19 \mathrm{~d} \\
0.03\end{array}$ & $\begin{array}{l}0.14 \mathrm{a} \\
\pm 0.04\end{array}$ & $\begin{array}{l}0.17 \mathrm{c} \\
\pm 0.03\end{array}$ & $\begin{array}{l}0.17 \mathrm{c} \\
\pm 0.01\end{array}$ \\
\hline $\begin{array}{l}\text { HO-1 } \\
\text { gene } \\
(24 \mathrm{H})\end{array}$ & $\begin{array}{l}\text { Mean } \\
\pm \mathrm{SD}\end{array}$ & $\begin{array}{l}0.150 \mathrm{a} \\
\pm 0.05\end{array}$ & $\begin{array}{l}0.13 \mathrm{a} \\
\pm 0.02\end{array}$ & $\begin{array}{l}0.58 \mathrm{~b} \\
\pm 0.28\end{array}$ & $\begin{array}{l}0.89 \mathrm{c} \\
\pm 0.13\end{array}$ & $\begin{array}{l}0.55 \mathrm{~b} \\
\pm 0.16\end{array}$ & $\begin{array}{l}0.53 \mathrm{~b} \\
\pm 0.3\end{array}$ & $\begin{array}{l}0.98 \mathrm{c} \\
\pm 0.01\end{array}$ \\
\hline $\begin{array}{l}\text { HO-1 } \\
(1 \mathrm{WK})\end{array}$ & $\begin{array}{l}\text { Mean } \\
\pm \text { SD }\end{array}$ & $\begin{array}{l}0.146 \mathrm{a} \\
\pm 0.05\end{array}$ & $\begin{array}{r}0.12 \mathrm{a} \\
\pm 0.02 \\
\end{array}$ & $\begin{array}{l}0.53 \mathrm{~b} \\
\pm 0.14\end{array}$ & $\begin{array}{l}0.86 \mathrm{c} \\
\pm 0.17\end{array}$ & $\begin{array}{l}0.53 b \\
\pm 0.17\end{array}$ & $\begin{array}{r}0.41 \mathrm{~b} \\
\pm 0.12 \\
\end{array}$ & $\begin{array}{l}0.98 \mathrm{c} \\
\pm 0.01\end{array}$ \\
\hline
\end{tabular}

Table (2): Statistical ANOVA one way of HO-1 gene:

\begin{tabular}{|c|c|c|c|c|}
\hline $\begin{array}{c}\text { HO-1 gene } \\
\text { Depend. } \\
\text { Variable }\end{array}$ & $\begin{array}{c}\text { Sum of } \\
\text { Squares }\end{array}$ & $\begin{array}{c}\text { Mean } \\
\text { Square }\end{array}$ & F & P value \\
\hline Group & 12.346 & 1.543 & 120.502 & 0.001 \\
\hline Period & 9.285 & 4.643 & 362.511 & 0.001 \\
\hline Group\#Period & 5.582 & 0.349 & 27.241 & 0.001 \\
\hline
\end{tabular}

\section{b. Change in HO-1 enzyme activity}

Results showed that there was a marked decrease in $\mathrm{HO}$ enzyme activity in diabetic rat group. Administration of either natural curcumin, NCD, sildenafil, or sildenafil combined with NCD led to a significant elevation in the enzyme activity in comparison to diabetic group that persisted up to $24 \mathrm{hr}$ with all of the above mentioned treatment protocols and up to 5 days with NCD and combined NCD + Tadalafile Combined NCD with Tadalafile showed significant enhancing when compared to sildenafil alone (Tables $3 \& 4$ ).

Table (3): HO-1 enzyme activity in rats treated with curcumin, tadalafil after injection with streptozotocinat different periods :

\begin{tabular}{|c|c|c|c|c|c|c|c|}
\hline Groups & $\begin{array}{l}\text { Mean } \\
\pm \mathrm{SD}\end{array}$ & Control & DM & $\begin{array}{l}\text { DM+ } \\
\text { p.Curc. }\end{array}$ & $\begin{array}{l}\text { DM+ } \\
\text { Curc.D. }\end{array}$ & $\begin{array}{l}\text { DM+ } \\
\text { Tada. }\end{array}$ & $\begin{array}{l}\text { DM+ } \\
\text { Tada.+ } \\
\text { Curc.D }\end{array}$ \\
\hline $\begin{array}{l}\text { HO-1 } \\
(1.5 \mathrm{H})\end{array}$ & $\begin{array}{l}\text { Mean } \\
\pm S D\end{array}$ & $\begin{array}{l}15.00 \mathrm{~cd} \\
\pm 1.41\end{array}$ & $\begin{array}{l}7.00 \mathrm{a} \\
\pm 1.94\end{array}$ & $\begin{array}{l}12.00 \mathrm{~b} \\
\pm 1.05\end{array}$ & $\begin{array}{l}17.50 \mathrm{e} \\
\pm 1.18\end{array}$ & $\begin{array}{l}19.00 \mathrm{c} \\
\pm 1.25\end{array}$ & $\begin{array}{l}19.00 \mathrm{~d} \\
\pm 2.91\end{array}$ \\
\hline $\begin{array}{l}\mathrm{HO}-1 \\
(24 \mathrm{H})\end{array}$ & $\begin{array}{l}\text { Mean } \\
\pm \mathrm{SD}\end{array}$ & $\begin{array}{l}14.70 \mathrm{c} \\
\pm 1.40\end{array}$ & $\begin{array}{l}7.00 \mathrm{a} \\
\pm 1.94\end{array}$ & $\begin{array}{l}10.00 \mathrm{~b} \\
\pm 1.15\end{array}$ & $\begin{array}{l}15.00 \mathrm{c} \\
\pm 1.15\end{array}$ & $\begin{array}{l}14.90 \mathrm{c} \\
\pm 2.47\end{array}$ & $\begin{array}{l}17.00 \mathrm{c} \\
\pm 2.31\end{array}$ \\
\hline $\begin{array}{l}\text { HO-1 } \\
(1 \mathrm{WK})\end{array}$ & $\begin{array}{l}\text { Mean } \\
\pm S D\end{array}$ & $\begin{array}{l}15.10 \mathrm{~d} \\
\pm 1.42\end{array}$ & $\begin{array}{r}7.00 \mathrm{a} \\
\pm 1.94\end{array}$ & $\begin{array}{l}7.40 \mathrm{~b} \\
\pm 1.65\end{array}$ & $\begin{array}{l}9.50 \mathrm{~b} \\
\pm 1.08\end{array}$ & $\begin{array}{l}7.40 \mathrm{~b} \\
\pm 1.78\end{array}$ & $\begin{array}{l}12.00 \mathrm{c} \\
\pm 2.01\end{array}$ \\
\hline
\end{tabular}


Table (4): Statistical ANOVA one way of HO-1 enzyme:

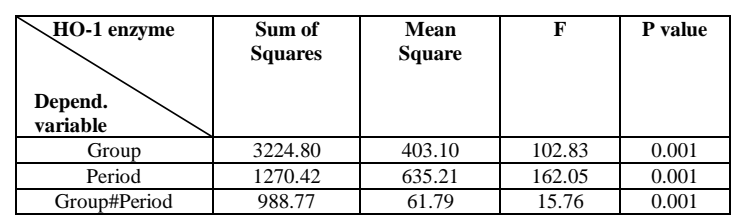

\section{c. Change in cGMP level}

Results showed that there was a significant decrease in cGMP levels in the diabetic group. Administration of either curcumin, NCD or Tadalafile or combinations of NCD with Tadalafile led to a significant elevation of cGMP levels that persisted up to one week in comparison to the diabetic group. Administration of Tadalafile alone led to a significant elevation of cGMP levels in comparison to the diabetic group that persisted for $1 \mathrm{hr}$ (Tables 5\&6).

Table (5): Means of Tissue cGMP enzyme activity in rats treated with curcumin, tadalafil after injection with streptozotocinat different period s :

\begin{tabular}{|c|c|c|c|c|c|c|c|}
\hline Groups & Mean & & & DM+ & DM+ & DM+ & DM+ Tada. \\
Period & $\begin{array}{c} \pm \\
\text { SD }\end{array}$ & Control & DM & p.Curc. & Curc.D. & Tada. & Curc.D \\
\hline cGMP & Mean & $3.53 \mathrm{a}$ & $0.50 \mathrm{~b}$ & $4.00 \mathrm{bc}$ & $4.90 \mathrm{bcd}$ & $4.00 \mathrm{~d}$ & $5.20 \mathrm{~d}$ \\
$(1.5 \mathrm{H})$ & \pm & \pm & \pm & \pm & \pm & \pm & \pm \\
\hline cGMP & SD & 0.85 & 0.13 & 0.82 & 1.1 & 0.67 & 1.72 \\
$(24 \mathrm{H})$ & \pm & \pm & \pm & \pm & \pm & \pm & $\pm .20 \mathrm{c}$ \\
\hline & SD & 0.88 & 0.13 & 0.82 & 1.1 & 0.94 & 1.72 \\
\hline cGMP & Mean & $3.55 \mathrm{a}$ & $0.50 \mathrm{~b}$ & $4.10 \mathrm{ca}$ & $5.20 \mathrm{~d}$ & $0.52 \mathrm{cb}$ & $5.00 \mathrm{~d}$ \\
$(1 \mathrm{WK})$ & \pm & \pm & \pm & \pm & \pm & \pm & \pm \\
\hline
\end{tabular}

Table (6): Statistical a nova one way of cGMP enzyme:

\begin{tabular}{|c|c|c|c|c|}
\hline $\begin{array}{c}\text { cGMP } \\
\text { enzyme }\end{array}$ & $\begin{array}{c}\text { Sum of } \\
\text { Squares } \\
\text { variable }\end{array}$ & $\begin{array}{c}\text { Mean } \\
\text { Square }\end{array}$ & F & P value \\
\hline Group & 242.09 & 0.98 & 20.57 & $<0.001$ \\
\hline Period & 19.50 & 0.72 & .001 & 1.00 \\
\hline Group\#Period & 390.6 & 24.41 & 1.113 & .343 \\
\hline
\end{tabular}

\section{d. change in NOS enzyme activity}

Results showed that there was a significant decrease in NOS enzyme activity levels in the diabetic group. There was a significant elevation in NOS activity levels in all treated rat groups that persisted up to 5 days in comparison to the untreated diabetic group (with curcumin, NCD, Tadalafile, NCD combined with Tadalafile). Administration of Tadalafile or its combinations with NCD led to a significant elevation in NOS levels after $24 \mathrm{hr}$ in comparison to NCD rat group (Tables7\&8).
Table (7): NOS enzyme activity in rats treated with curcumin, tadalafil after injection with streptozotocinat different periods :

\begin{tabular}{|c|c|c|c|c|c|c|c|}
\hline Period & $\begin{array}{c}\text { Mean } \\
\\
\stackrel{ \pm}{\mathrm{SD}}\end{array}$ & Control & DM & $\begin{array}{l}\text { DM+ } \\
\text { p.Curc. }\end{array}$ & $\begin{array}{l}\text { DM+ } \\
\text { Curc.D. }\end{array}$ & $\begin{array}{l}\text { DM+ } \\
\text { Tada. }\end{array}$ & $\begin{array}{l}\text { DM+ } \\
\text { Tada.+ } \\
\text { Curc.D } \\
\end{array}$ \\
\hline $\begin{array}{l}\text { NOS } \\
(1.5 \mathrm{H})\end{array}$ & $\begin{array}{c}\text { Mean } \\
\pm \\
\text { SD } \\
\end{array}$ & $\begin{array}{c}892.0 \mathrm{~b} \\
\pm \\
60.05 \\
\end{array}$ & $\begin{array}{c}726.0 \mathrm{a} \\
\pm \\
17.03 \\
\end{array}$ & $\begin{array}{c}1191.0 \mathrm{c} \\
\pm \\
91.55\end{array}$ & $\begin{array}{c}1218.0 \mathrm{~cd} \\
\pm \\
66.51 \\
\end{array}$ & $\begin{array}{c}1160.0 \mathrm{c} \\
\pm \\
74.58 \\
\end{array}$ & $\begin{array}{c}1381.0 \mathrm{e} \\
\pm \\
151.1 \\
\end{array}$ \\
\hline $\begin{array}{l}\text { NOS } \\
(24 \mathrm{H})\end{array}$ & $\begin{array}{c}\text { Mean } \\
\pm \\
\text { SD }\end{array}$ & $\begin{array}{c}\text { 894.0ab } \\
\quad \pm \\
61.05 \\
\end{array}$ & $\begin{array}{c}726.0 \mathrm{a} \\
\quad \\
17.03 \\
\end{array}$ & $\begin{array}{c}1078.0 \mathrm{bc} \\
\pm \\
45 \\
\end{array}$ & $\begin{array}{c}1300.0 \mathrm{~cd} \\
\pm \\
80.73 \\
\end{array}$ & $\begin{array}{c}1413.2 \mathrm{de} \\
\pm \\
486.93 \\
\end{array}$ & $\begin{array}{c}1790.0 \mathrm{f} \\
\pm \\
322.33 \\
\end{array}$ \\
\hline $\begin{array}{l}\text { NOS } \\
\text { (1WK) }\end{array}$ & $\begin{array}{c}\text { Mean } \\
\pm \\
\text { SD }\end{array}$ & $\begin{array}{c}812.0 \mathrm{a} \\
\pm \\
47.56\end{array}$ & $\begin{array}{c}726.0 \\
\pm \\
39.73\end{array}$ & $\begin{array}{c}1096.30 \mathrm{~b} \\
\pm \\
52.04\end{array}$ & $\begin{array}{c}1181.0 \mathrm{~b} \\
\pm \\
75.02\end{array}$ & $\begin{array}{c}1180.0 \mathrm{~b} \\
\pm \\
74.21\end{array}$ & $\begin{array}{c}1280.0 \mathrm{~b} \\
\pm \\
290.52\end{array}$ \\
\hline
\end{tabular}

Table (8): Statistical a nova one way of NOS enzyme:

\begin{tabular}{|c|c|c|c|c|}
\hline $\begin{array}{l}\text { NOS enzyme } \\
\text { Depend. } \\
\text { variable }\end{array}$ & $\begin{array}{c}\text { Sum of } \\
\text { Squares }\end{array}$ & $\begin{array}{c}\text { Mean } \\
\text { Square }\end{array}$ & F & P value \\
\hline Group & 3224.80 & 403.10 & 102.83 & 0.001 \\
\hline Period & 1270.42 & 635.21 & 162.05 & 0.001 \\
\hline Group\#Period & 988.77 & 61.79 & 15.76 & 0.001 \\
\hline
\end{tabular}

\section{e. Change in ICP}

Results showed that there was a significant decrease in ICP/MAP in the diabetic rat group. There was a significant progressive elevation in ICP/MAP that rises with increasing the frequency of electric current stimulation in all treated diabetic rat groups with all treatment regimens in comparison with untreated diabetic rat group. NCD showed superior effects in comparison to curcumin at 0.3 and 0.5 $\mathrm{Hz}$ (Tables 9\&10).

\section{f. Change in NF - $\beta$}

Results showed that there was a significant elevation in $\mathrm{NF}-\beta$ in the diabetic group. There was a significant decrease in NF $-\beta$ in all treated diabetic rat groups with all treatment regimens that persisted up to 5 days in comparison to untreated diabetic group except with Tadalafile group where the effect persisted for $1 \mathrm{hr}$ (Table 11\&12).

\section{g. Change in P38}

Results showed that there was a significant increase in p38 gene expression in the diabetic group. There was a significant decrease in p38 gene that persisted up to $24 \mathrm{hr}$ in the diabetic rat groups treated with curcumin, NCD, NCD combinations with Tadalafile in comparison to the untreated diabetic group (Table 13\&14). 
Table (9): ICP in rats treated with curcumin, tadalafil after injection with streptozotocinat different periods :

\begin{tabular}{|c|c|c|c|c|c|c|c|}
\hline Period & Mean \pm SD & Control & DM & DM+ p.Curc. & DM+ Curc.D. & DM+ Tada. & DM+ Tada.+ Curc. D \\
\hline $\mathrm{ICP}(0.3 \mathrm{HZ})$ & $\begin{array}{c}\text { Mean } \\
\pm \\
\text { SD }\end{array}$ & $\begin{array}{c}1.00 \mathrm{ab} \\
\pm \\
0.41\end{array}$ & $\begin{array}{c}.50 \mathrm{a} \\
\pm \\
0.23\end{array}$ & $\begin{array}{c}1.50 \mathrm{bc} \\
\pm \\
0.35\end{array}$ & $\begin{array}{c}2.20 \mathrm{~cd} \\
\pm \\
0.73\end{array}$ & $\begin{array}{c}2.00 \mathrm{~cd} \\
\pm \\
0.76\end{array}$ & $\begin{array}{c}2.20 \mathrm{~cd} \\
\pm \\
0.69\end{array}$ \\
\hline $\mathrm{ICP}(0.5 \mathrm{HZ})$ & $\begin{array}{c}\text { Mean } \\
\pm \\
\text { SD }\end{array}$ & $\begin{array}{c}2.20 \mathrm{ab} \\
\pm \\
0.73\end{array}$ & $\begin{array}{c}1.20 \mathrm{a} \\
\pm \\
0.47\end{array}$ & $\begin{array}{c}2.30 \mathrm{ab} \\
\pm \\
0.66\end{array}$ & $\begin{array}{c}3.50 \mathrm{c} \\
\pm \\
0.91\end{array}$ & $\begin{array}{c}3.20 \mathrm{bc} \\
\pm \\
1.1\end{array}$ & $\begin{array}{c}3.70 \mathrm{c} \\
\pm \\
0.97\end{array}$ \\
\hline $\mathrm{ICP}(0.8 \mathrm{HZ})$ & $\begin{array}{c}\text { Mean } \\
\pm \\
\text { SD }\end{array}$ & $\begin{array}{c}4.50 \mathrm{bc} \\
\pm \\
1.63\end{array}$ & $\begin{array}{c}2.38 \mathrm{a} \\
\pm \\
0.62\end{array}$ & $\begin{array}{c}4.20 \mathrm{abc} \\
\pm \\
1.22\end{array}$ & $\begin{array}{c}5.10 \mathrm{c} \\
\pm \\
0.91\end{array}$ & $\begin{array}{c}5.00 \mathrm{c} \\
\pm \\
2.05\end{array}$ & $\begin{array}{c}5.50 \mathrm{c} \\
\pm \\
0.47\end{array}$ \\
\hline ICP (1 HZ) & $\begin{array}{c}\text { Mean } \\
\pm \\
\text { SD }\end{array}$ & $\begin{array}{c}5.00 \mathrm{abc} \\
\pm \\
2.05\end{array}$ & $\begin{array}{c}2.80 \mathrm{a} \\
\pm \\
0.93\end{array}$ & $\begin{array}{c}5.50 \mathrm{bc} \\
\pm \\
2.17\end{array}$ & $\begin{array}{c}6.00 \mathrm{bc} \\
\pm \\
2.05\end{array}$ & $\begin{array}{c}6.50 \mathrm{c} \\
\pm \\
1.84\end{array}$ & $\begin{array}{c}6.60 \mathrm{c} \\
\pm \\
2.27\end{array}$ \\
\hline
\end{tabular}

Table (10): Statistical a nova one way of ICP :

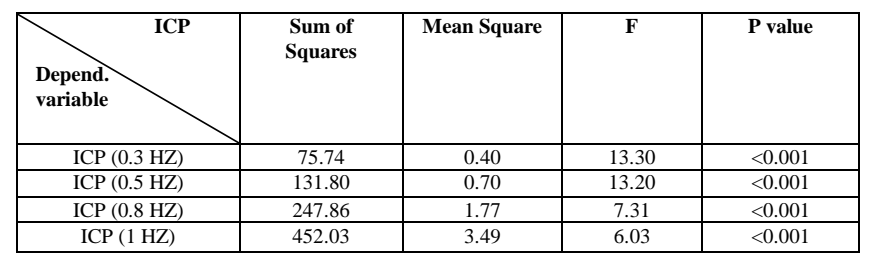

Table (11): NF $-\beta$ Gene in rats treated with curcumin, tadalafil after injection with streptozotocinat different periods :

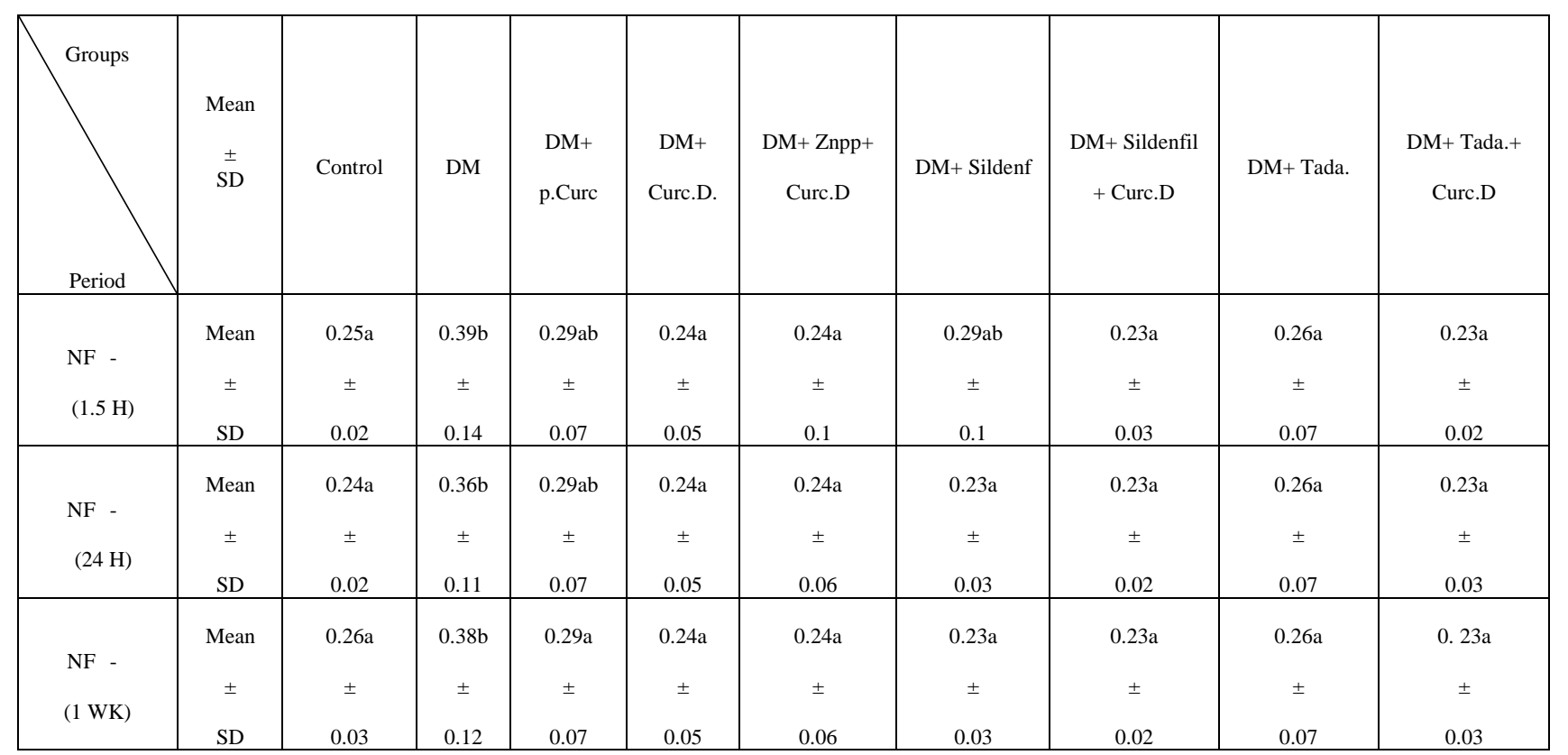


Table (12): Statistical ANOVA one way of NF $-\beta$ gene :

\begin{tabular}{|c|c|c|c|c|}
\hline $\begin{array}{l}\text { Depend. } \\
\text { variable }\end{array}$ & $\begin{array}{c}\text { Type III Sum } \\
\text { of Squares }\end{array}$ & Mean Square & F & P value \\
\hline Group & 0.52 & 0.06 & 16.19 & 0.001 \\
\hline Period & 0.01 & 0.01 & 0.27 & $\mathbf{0 . 7 6}$ \\
\hline Group\#Period & 0.01 & 0.01 & 0.27 & 0.76 \\
\hline
\end{tabular}

Table (13): p38 Gene in rats treated with curcumin , tadalafil after injection with streptozotocinat different periods :

\begin{tabular}{|c|c|c|c|c|c|c|c|}
\hline Groups & Mean & & & & & & DM+ \\
Period & & Control & DM & DM+ & DM+ & DM+ & Tada.+ \\
\hline P38 & Mean & $0.13 \mathrm{a}$ & $0.29 \mathrm{~b}$ & $0.18 \mathrm{a}$ & $0.15 \mathrm{a}$ & $0.28 \mathrm{~b}$ & $0.16 \mathrm{a}$ \\
$(1.5$ & $\pm \mathrm{SD}$ & \pm 0.06 & \pm 0.07 & \pm 0.08 & \pm 0.03 & \pm 0.06 & \pm 0.03 \\
$\mathrm{H})$ & Curc.D & Tada. & \\
\hline $\mathrm{P} 38$ & Mean & $0.12 \mathrm{a}$ & $0.29 \mathrm{~b}$ & $0.20 \mathrm{a}$ & $0.16 \mathrm{a}$ & $0.30 \mathrm{~b}$ & $0.17 \mathrm{a}$ \\
$(24 \mathrm{H})$ & $\pm \mathrm{SD}$ & \pm 0.06 & \pm 0.07 & \pm 0.05 & \pm 0.03 & \pm 0.08 & \pm 0.07 \\
\hline $\mathrm{P} 38$ & Mean & $0.14 \mathrm{a}$ & $0.29 \mathrm{~b}$ & $0.26 \mathrm{~b}$ & $0.24 \mathrm{~b}$ & $0.29 \mathrm{~b}$ & $0.26 \mathrm{~b}$ \\
$(1 \mathrm{WK})$ & $\pm \mathrm{SD}$ & \pm 0.06 & \pm 0.07 & \pm 0.05 & \pm 0.05 & \pm 0.07 & \pm 0.05 \\
\hline
\end{tabular}

Table (14): Statistical ANOVA one way of 38 gene:

\begin{tabular}{|c|c|c|c|c|}
\hline $\begin{array}{l}\text { P38 gene } \\
\text { Dependent } \\
\text { Variable }\end{array}$ & $\begin{array}{c}\text { Sum of } \\
\text { Squares }\end{array}$ & $\begin{array}{c}\text { Mean } \\
\text { Square }\end{array}$ & F & P value \\
\hline Group & 0.78 & 0.09 & 32.96 & 0.001 \\
\hline Period & 0.14 & 0.07 & 23.72 & $\mathbf{0 . 0 0 1}$ \\
\hline Group\#Period & 0.11 & 0.01 & 2.37 & 0.003 \\
\hline
\end{tabular}

\section{h. Change in Nrf2}

Results showed that there was a significant increase in Nrf2 gene expression in diabetic rat groups treated with curcumin, NCD, combinations of NCD with Tadalafile in comparison to the untreated diabetic rat group and the control group (Table 15\&16).

Table (15): Nrf2 Gene in rats treated with curcumin, tadalafil after injection with streptozotocinat different periods :

\begin{tabular}{|c|c|c|c|c|c|c|c|}
\hline Groups & Mean & & & & & & DM+ \\
Period & & Control & DM & DM+ & DM+ & DM+ & Tada.+ \\
\hline Nrf2 & Mean & $0.251 \mathrm{a}$ & $0.25 \mathrm{a}$ & $0.35 \mathrm{ab}$ & $0.42 \mathrm{~b}$ & $0.26 \mathrm{a}$ & $0.42 \mathrm{~b}$ \\
$(1.5 \mathrm{H})$ & $\pm \mathrm{SD}$ & \pm 0.03 & \pm 0.04 & \pm 0.11 & \pm 0.06 & \pm 0.05 & \pm 0.07 \\
\hline Nrf2 & Mean & $0.254 \mathrm{a}$ & $0.25 \mathrm{a}$ & $0.37 \mathrm{~b}$ & $0.45 \mathrm{~b}$ & $0.27 \mathrm{a}$ & $0.45 \mathrm{~b}$ \\
$(24 \mathrm{H})$ & $\pm \mathrm{SD}$ & \pm 0.03 & \pm 0.04 & \pm 0.09 & \pm 0.06 & \pm 0.05 & \pm 0.06 \\
\hline Nrf2 & Mean & $0.252 \mathrm{a}$ & $0.25 \mathrm{a}$ & $0.36 \mathrm{bc}$ & $0.43 \mathrm{c}$ & $0.26 \mathrm{ab}$ & $0.43 \mathrm{c}$ \\
$(1 \mathrm{WK})$ & $\pm \mathrm{SD}$ & \pm 0.03 & \pm 0.04 & \pm 0.11 & \pm 0.07 & \pm 0.05 & \pm 0.06 \\
\hline
\end{tabular}

Table (16): Statistical ANOVA one way of Nrf2 gene:

\begin{tabular}{|c|c|c|c|c|}
\hline $\begin{array}{c}\text { Nrf2 gene } \\
\text { Dariablent }\end{array}$ & Sum of Squares & $\begin{array}{c}\text { Mean } \\
\text { Square }\end{array}$ & F & $\begin{array}{c}\mathbf{P} \\
\text { val } \\
\text { ue }\end{array}$ \\
\hline Group & 1.66 & 0.20 & 43.81 & $\begin{array}{c}0.0 \\
01\end{array}$ \\
\hline Period & 0.02 & 0.01 & 3.07 & $\begin{array}{c}\mathbf{0 . 0} \\
4\end{array}$ \\
\hline Group\#Period & 0.17 & 0.01 & 2.29 & $\begin{array}{c}0.0 \\
4\end{array}$ \\
\hline
\end{tabular}

\section{Discussion}

Erectile response depends on nitric oxide (NO) generated by NO synthase (NOS) enzyme of the nerves and vascular endothelium in the cavernous tissue. NO activates soluble guanylate cyclase ( $\mathrm{sGC}$ ), leading to the production of cyclic guanosine monophosphate (cGMP) which activates cGMP-dependent protein kinase that activates $\mathrm{Ca}^{2+} /$ ATPase pump with subsequent activation of $\mathrm{Ca}^{2+} / \mathrm{K}$ efflux pump extruding $\mathrm{Ca}^{2+}$ across the plasma membrane resulting in smooth muscle cell relaxation. A role similar to that of NOS/NO signaling has been proved for carbon monoxide (CO) produced in mammals from heme catabolism by heme oxygenase (HO) enzyme (Abdel Aziz et al., 2009). The concept that HO-derived $\mathrm{CO}$ could play a role in mediating erectile function acting in synergism with, or as a potentiator for, NOS/NO signaling pathway is gaining momentum. $\mathrm{CO} / \mathrm{HO}$ signaling pathway has been shown to partially mediate the actions of oral phosphodiesterase-5 inhibitors (PDE5 Is). (Abdel aziz et al. 2007, 2008) reported that the effect of the three available PDE5 Is; sildenafil, verdenafil and tadalafil are partially mediated via upregulation of $\mathrm{HO}$ enzyme activity and their effect is partially inhibited following administration of $\mathrm{HO}$ inhibitor. Results showed that administration of all drug regimen interventions led to a significant increase in ICP/MAP as compared to the diabetic group. The present result is in agreement with other reported, showed the Endothelial dysfunction is the main a etiologic factor of vasculogenic erectile dysfunction (ED) (Strong TD et al., 2008). In diabetes hyperglycemia affects endothelial functions by synthesis of growth factors and vasoactive agents (Yang et al., 2010). Hyperglycemia activate protein kinase-C (PKC) that induces phosphorylation of p115RhoGEF, a guanine nucleotide exchange factor for Rho GTPase (Xu et al., 2009, Chen et al., 2005). Active RhoA is implicated in arginase induction leading to decrease in nitric oxide (NO) bioavailability (Romero et al., 2008, Abdel Aziz et al., 2009). Furthermore, activated PKC leads to sustained increases in the production of superoxide anion $(\mathrm{O} 2 \bullet-)$ that activates $N F-\beta$ and affecting the expression of endothelial NOS (Enos) (Zhang et al., 2008). Biochemical Results by Elisa method showed revealed that there were significant decreases in cavernous tissue cGMP, NOS and hemeoxygenase enzymatic activities in diabetic rats as compared to control rats. These results is agreement with others who found (Wang et al., 
2003, Abdel Aziz et al., 2009) who reported that administration of either curcumin, NCD, Tadalafile or Tadalafile combined with NCD led to significant elevations in cGMP levels, NOS and HO-1 cavernous tissue enzyme levels with significantly higher sustained effect in favor of NCD. The molecular study by Real time RT PCR.the gene expression profile of NF $-\beta$ and p38 were significantly increased in STZ-induced diabetic rat. Administration of either natural curcumin or NCD led to a significant lowering effect on their gene expression with more significant superior effects with NCD. Nrf2 gene expression was unchanged in STZ-induced diabetic rats, whereas its levels were significantly elevated with curcumin and NCD. In concordant with these results (Aggarwal et al.,2009) reported stated that curcumin suppresses NF $-\beta$ and activates Nrf2 cell-signaling pathways. Reported that $\mathrm{HO}$ protects $\mathrm{NO}$ through scavenging of reactive oxygen species (ROS), preventing the formation of peroxynitrite and subsequent degradation of NO. (Ahmad et al.,2009) stated that over expression of HO-1 may mediate an increase in eNOS and a decrease in iNOS, with restoration of vascular responses in diabetic rats. Curcumin as an inducer of HO-1 could indirectly potentiate eNOS effects on vascular endothelium. (Williams et al.,2005) stated that CO like NO, acts as a vasorelaxant. $\mathrm{HO}-1$-derived $\mathrm{CO}$ has a positive effect on both sGC and cGMP levels in vascular endothelial cells (Aggarwal et al., 2009).

In conclusion, NCD could enhance erectile function in a diabetic model via up-regulation of cavernous tissue levels of HO-1 gene and cGMP. NCD is superior to curcumin with more prolonged duration of action.

\section{References}

Abdel Aziz, M.T., Mostafa, T., Atta, H.; Rashed, L., Marzouk, SA., Ohaia, EM., Sabry, D., Hassona, A.A., EIShehaby, A.M. and Abdel Aziz, A.T (2007). Oral phosphodiesterase-5 inhibitors: effect of heme oxygenase inhibition on cGMP signalling in rat cavernous tissue. Andrologia, 39 (2): 66-70.

Abdel Aziz, M.T., Abraham, N., Mostafa, T., Mahfouz, S., Atta, H., Wassef, M.A., Fouad, H., Rashed, L..A., Sabry, D. and AbdEl Azim, O. (2008). Heme oxygenase-1 (HO-1) gene transfer and CORM-3 as therapeutic modalities in erectile dysfunction. Andrologia, 5: $363-43$.

Abdel Aziz, M.T., El Asmer, M.F., Mostafa ,T., Atta, H., Mahfouz, S. and Fouad, H. (2009). Effects of losartan, HO-1 inducers or HO-1 inhibitors on erectile signaling in diabetic rats. J. Sex. Med., 6: 3254-3264.

Abdel Aziz, M.T., Motawi, T., Rezq, A., Mostafa, T., Fouad, H.H. and Ahmed H.H. (2012). Effects of a watersoluble curcumin protein conjugate vs. pure curcumin in a diabetic model of erectile dysfunction. J Sex Med., 9: 1815-1833.

Aggarwal, B.B., Kumar, A. and Bharti, A.C. (2003). Anticancer potential of Curcumin: Preclinical and clinical studies. Anticancer Res., 23: 363-98.
Aggarwal. B.B. and Harikuma.r K.B. (2009). Potential therapeutic effects of curcumin, the anti-inflammatory agent, against neurodegenerative, cardiovascular, pulmonary, metabolic, autoimmune and neoplastic diseases. Int. J. Biochem. Cell Biol., 41(1):40-59.

Chen, Z., Singer, W.D., Sternweis, P.C. and Sprang, S.R. (2005). Structure of the p115RhoGEF rgRGS domainGalpha13/i1 chimera complex suggests convergent evolution of a GTPase activator. Nat. Struct. Mol. Biol., 2: 191-197.

Hamed, S., Brenner, B. and Roguin, A. (2011). Nitric Oxide: a Key Factor behind the Dysfunctionality of Endothelial Progenitor Cells in Diabetes Mellitus Type-2. Cardiovasc. Res., 91: 9-15.

Hatcher, H., Planalp, R., Cho, J., Torti, F.M. and Torti, S.V. (2008). Curcumin: from ancient medicine to current clinical trials. Cell. Mol. Life Sci., 65 (11): 1631-1652.

Holinstat, M., Mehta, D., Kozasa, T., Minshall, R.D. and Malik, A.B. (2003). Protein kinase Calpha-induced p115RhoGEF phosphorylation signals endothelial cytoskeletal rearrangement. J. Biol. Chem., 31: 2879328798.

King, H. and Rewers, M. (1998). Diabetes Reporting Group: Global estimates for prevalence of diabetes and impaired glucose tolerance in adults. Diabetes Care, 16:157-177.

Hu, J.J., Gu, H. Y., Ding, W. L., Yuan, S., Zhong, M. F. and Liu, L. (2008). Correlation between nNOS expression and erectile dysfunction in experimental diabetes mellitus rats of differents ages. Neuroembryol Aging, 5:144-50.

Li, W.J., Zbou, J., Li, B., Wang, H., Peng, Y.B. and Wang, Z. (2011). Inhibition restores erectile function by suppressing corporal smooth muscle apoptosis in diabetic rats. J. Sex Med., 8:1072-1082.

Lindberg, E., Theorell-Haglow, J., Svensson M., Gislason, T., Berne, C. and Janson, C. (2012). Sleep apnea and glucose metabolism - a long-term follow-up in a community-based sample. Chest. [Epub ahead of print]. 10.1378/chest.11-1844.

Mulhall, J.P., Verma, N., Deveci, S., Tal, R., Kobylarz, K. and Müller, A. (2013). Sildenafil citrate improves erectile function after castration in a rat model. B.J.U. Int.: 10.1111/bju.12175.

Romero, M.J., Platt, D.H., Tawfik, H.E., Labazi, M., El-Remessy, A.B. and Bartoli, M.(2008). Diabetes-induced coronary vascular dysfunction involves increased arginase activity. Circ. Res., 1: 95-102.

Seo, K.I., Choi, M.S. and Jung, U.J. (2008). Effect of curcumin supplementation on blood glucose.; plasma insulin.; and glucose homeostasis related enzyme activities in diabetic db/db mice. Mol. Nutr. Food Res., 52:995-1004.

Strong, T.D., Gebska, M.A., Burnett, A.L., Champion, H.C. and Bivalacqua, T.J.( 2008): Endothelium-specific gene and stem cell-based therapy for erectile dysfunction. Asian J. Androl.,10: 14-22.

Wang, J., Lu S., Moenne, P. and Montellano, O.R. (2003). Interaction of nitric oxide with human heme oxygenase-1.; J. Biol. Chem., 278(4): 2341 - 2347. 
Williams, B.A., Liu, C.; Deyoung, L., Brock, G.B. and Sims, S.M. (2005). Regulation of intracellular $\mathrm{Ca}^{2+}$ release in corpus cavernosum smooth muscle: Synergism between nitric oxide and cGMP. Am. J. Physiol. Cell Physiol., 288: C650-658.

Xia, Z.W., Zhou, W.P., Cui, W.J., Zhang, X.H., Shen, Q.X.and Li, Y.Z. (2004). Structure prediction and activity analysis of human heme oxygenase- 1 and its mutant. World J. Gastroenterol., 10: 2352-2356.

$\mathrm{Xu}$, J.and Zou, M.H. (2009). Molecular insights and therapeutic targets for diabetic endothelial dysfunction. Circulation, 120: 1266-1286.

Yang, G., Lucas, R., Caldwell, R., Yao. L., Romero, M.J. and Caldwell, R.W. (2010). Novel mechanisms of endothelial dysfunction in diabetes. J. Cardiovasc. Dis. Res., 1:59-63.

Zhang, C. (2008). The role of inflammatory cytokines in endothelial dysfunction. Basic Res. Cardiol., 103: 398406.

Zhang, C., Bao, W., Rong, Y., Yang, H., Bowers, K., Yeung, E. and Kiely, M. (2013). Genetic variants and the risk of gestational diabetes mellitus: A systematic review. Hum. Reprod. Update, 19 (4): 376-90. 